This is a preprint of a paper intended for publication in a journal or proceedings. Since changes may be made before publication, this preprint is made available with the understanding that it will not be cited or reproduced without the permission of the author.

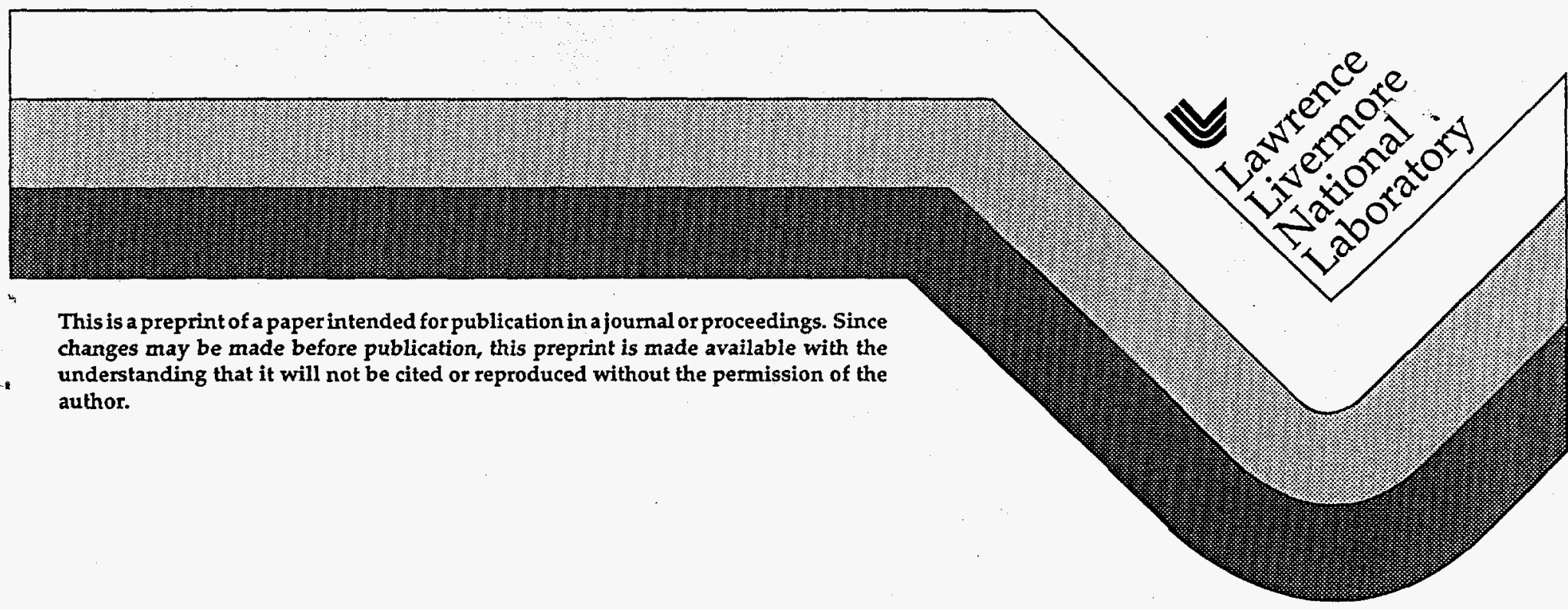




\section{DISCLAIMER}

This document was prepared as an account of work sponsored by an agency of the United States Government. Neither the United States Government nor the University of California nor any of their employees, makes any warranty, express or implied, or assumes any legal liability or responsibility for the accuracy, completeness, or usefulness of any information, apparatus, product, or process disclosed, or represents that its use would not infringe privately owned rights. Reference herein to any specific commercial product, process, or service by trade name, trademark, manufacturer, or otherwise, does not necessarily constitute or imply its endorsement, recommendation, or favoring by the United States Government or the University of California. The views and opinions of authors expressed herein do not necessarily state or reflect those of the United States Government or the University of California, and shall not be used for advertising or product endorsement purposes. 


\title{
Calculation of farfield distortion for a tilted-facet SOA
}

\author{
R. P. Ratowsky, S. Dijaili, J. Walker, F. Patterson, J. Kallman, and R. Deri \\ Lawrence Livermore National Laboratory \\ Livermore, CA 94551
}

\begin{abstract}
We explain the crescent-shaped farfield intensity distribution of tilted-facet semiconductor optical amplifiers (SOAs) using vector wave optics, and discuss implications for coupling to other optical elements.
\end{abstract}

Introduction. Semiconductor optical amplifiers (SOAs) are very important elements for telecommunications, computer communications, and signal processing applications. For stable, low noise operation, the modal reflection into the guided SOA mode must be minimized; modal reflectivity typically has to be kept below about $-40 \mathrm{~dB}$ [1]. This can be accomplished by antireflection (AR) coatings, or by tilting of the SOA end facet. The latter approach has been vigorously pursued recently, because effective AR coatings require very high tolerances and have polarization-dependent reflectivities.

Consequently, there has been a great deal of theoretical effort aimed at calculating the modal reflectivity from tilted interfaces, using a variety of approaches $[1,2]$.

However, there has been little attention directed toward calculating the transmitted field of a tilted-facet SOA. This is a problem of considerable importance, because the coupling of the SOA light to an element such as an optical fiber depends critically on the field distribution at the entrance plane to the fiber. Moreover, experimental measurements of the farfield of tilted-facet SOAs have revealed a curious crescent-shaped intensity distribution. To improve coupling efficiency it is important to understand to what extent this phenomenon is due to the SOA modal field distribution and to what extent it is due to the tilted interface.

Method. The SOA index and gain distribution (Fig. 1) result in a highly astigmatic nearfield distribution. From measurement, the nearfield intensity is found to be well approximated by a Gaussian $|\mathrm{E}(x, y)|^{2}=\exp \left(-2 x^{2} / \sigma_{x}^{2}-2 y^{2} / \sigma_{y}^{2}\right)$, with $\sigma_{\mathrm{x}} \approx 0.5 \mu \mathrm{m}$ and $\sigma_{\mathrm{y}}$ $\approx 5.0 \mu \mathrm{m}$, at a wavelength $\lambda=0.9 \mu \mathrm{m}$. Since the smaller dimension is of wavelength order, the light diverges very rapidly (nonparaxially). Thus, for accuracy we elect to use a full solution to Maxwell's equations for the propagtion. Our approach is based on a plane-wave expansion of the approximate SOA astigmatic Gaussian guided mode. Our procedure is as follows: We rotate to coordinates $x^{\prime}, y^{\prime}, z^{\prime}$, for which the $x^{\prime} y^{\prime}$ plane is in the plane of the tilted facet with tilt angle $\theta$ (see Fig. 2); we calculate the components of the field parallel and perpendicular to the plane of incidence ( $\mathrm{s}$ and $\mathrm{p}$ polarizations); we propagate the field through the tilted interface; finally, we rotate coordinates back to the original xyz system. 


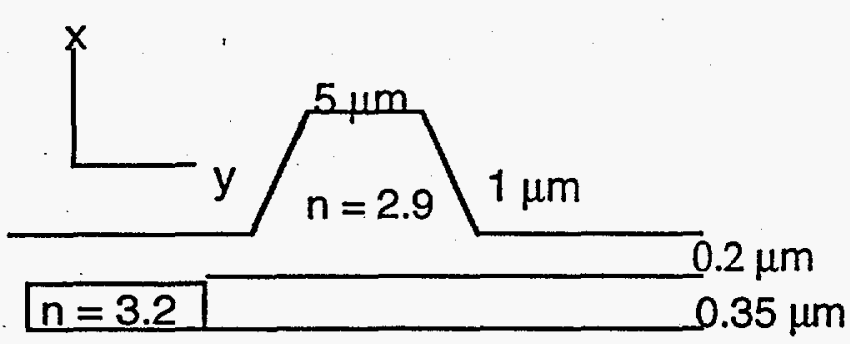

$\mathrm{n}=2.9$

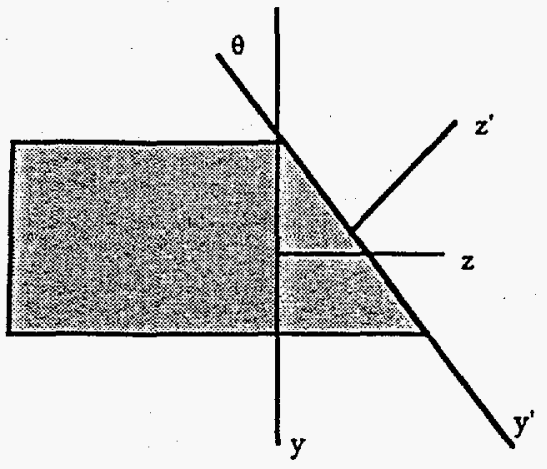

Fig. 2. Coordinates for tilted facet SOA

We make the approximation that the guided mode in the plane of the facet is given by the mode in the $x-y$ coordinates with a phase correction:

$$
E_{y}\left(x^{\prime}, y^{\prime} ; z^{\prime}=0\right)=\exp \left[i \beta_{0} y^{\prime} \sin (\theta)\right] E_{y}\left(x^{\prime}, y^{\prime} \cos (\theta)\right)
$$

where we are choosing TE polarization $\left(E_{x}=0\right)$.When this prescription is carried out, we find

$$
\begin{aligned}
& E_{x}=\sec \theta_{0} \iint d \alpha d \beta \bar{E}_{y}(\alpha, \beta) \frac{\alpha \beta}{\alpha^{2}+\beta^{2}}\left(T_{=}+T_{\perp}\right) \exp (i \psi) \\
& E_{y}=\sec \theta_{0} \iint d \alpha d \beta \bar{E}_{y}(\alpha, \beta) \frac{\alpha^{2} T_{=}+\beta^{2} T_{\perp}}{\alpha^{2}+\beta^{2}} \exp (i \psi) \\
& E_{z}=\sec \theta_{0} \iint d \alpha d \beta \bar{E}_{y}(\alpha, \beta) \frac{\beta T_{\equiv}}{\gamma} \exp (i \psi)
\end{aligned}
$$

where $\gamma=\sqrt{1-\alpha^{2}-\beta^{2}} ; T_{\perp}$ and $T_{=}$are the Fresnel transmission coefficents for $\mathrm{s}$ and $\mathrm{p}$ polarizations, respectively; $\psi=k(\alpha x+\beta y+\gamma) ; \bar{E}_{y}=\exp \left[\left(-k^{2} / 4\right)\left(\alpha^{2} \sigma_{x}^{2}+\beta^{2} \sigma_{y}^{2}\right)\right]$ and $\mathrm{k}=\omega / \mathrm{c}$.

Results. In Fig. 3 we show the result of our calculation of the SOA farfield intensity compared with the experimentally measured SOA farfield. The tilt angle $\theta=70$. Evidently the physics of Fresnel transmission is all that is required to explain the physics of the crescent shape. 

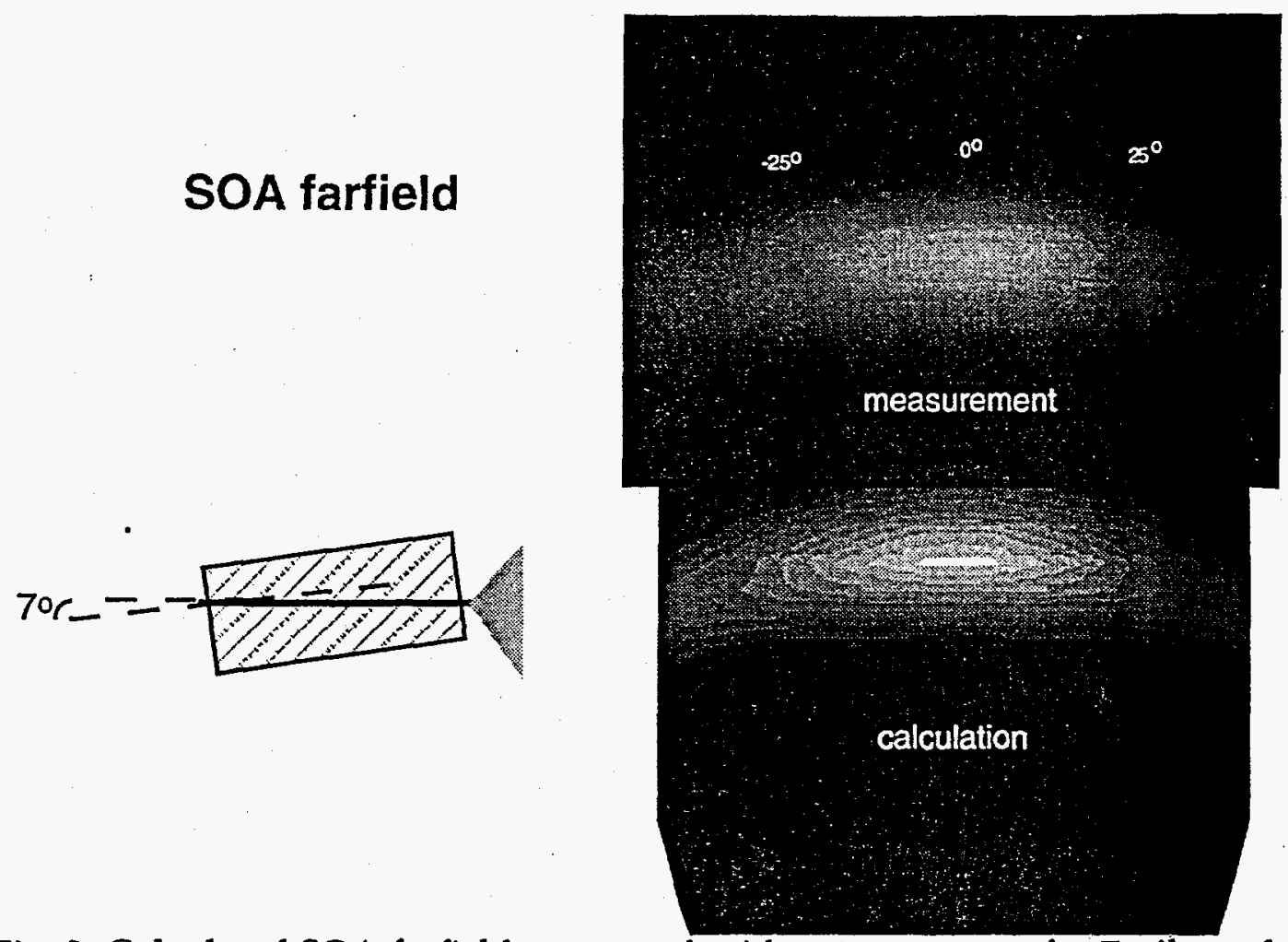

Fig. 3. Calculated SOA farfield compared with measurement for $7^{\circ}$ tilt angle.

To understand this effect further, it is worthwhile to consider the ray optics farfield one would expect for propagation of a highly astigmatic Gaussian through a tilted interface. We have carried out this calculation, and found the following result for the (scalar) farfield field distribution:

$$
\begin{aligned}
E(\alpha, \beta)= & \exp \left(-0.025 \alpha^{2}-.25 \tilde{\beta}^{2}\right) \\
\tilde{\beta}(\alpha, \beta)= & \beta \cos ^{2} \theta+\left(n^{2}-\alpha^{2}-\beta^{2}\right)^{\frac{1}{2}} \sin \theta \cos \theta \\
& -\left(1-\alpha^{2}-\left[\beta \cos \theta+n^{2}-\alpha^{2}-\beta^{2} \sin \theta\right]\right)^{\frac{1}{2}} \sin \theta
\end{aligned}
$$

This result is plotted in Fig. 4, using the coordinates $(\alpha, \beta)=\left(\sin \theta_{x}, \sin \theta_{y}\right)$, and we can clearly see that the ray optics picture does indeed capture the physics of the crescent shape, through coupling of the $\alpha$ and $\beta$ degrees of freedom. 


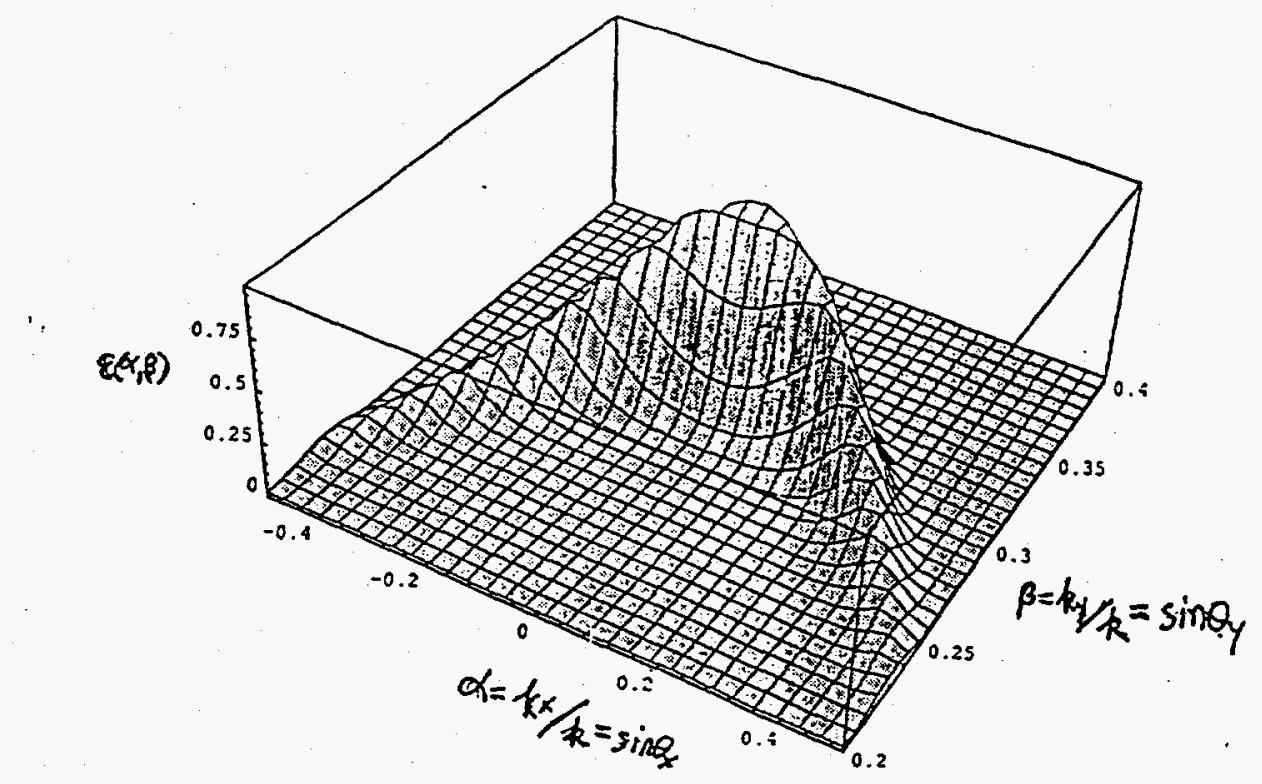

Fig. 4. Ray optics farfield for tilted-facet SOA

Since the farfield distortion is due to the tilted facet, we may improve the coupling efficiency from SOA to fiber by simply reversing the phase distortion. For example, if the light is coupled into an AR-coated semiconductor prism, the additional phase shift will approximately remove the phase distortion in the nearfield which produced the crescent-shape in the farfield. Moreover, if this correction is implemented in a manner similar to an anamorphic prism, the beam ellipticity can be reduced. Another approch might be to use an off-axis ball lens to compensate for the distortion while coupling to fiber; our ball-lens Maxwell solver code will allow us to model this configuration very accurately. In future calculcations we expect to demonstrate improved coupling to the crescent-shaped field by simpler techniques such as off-axis conically polished fibers, lensed fibers, etc.

Acknowledgement. This work was performed under the auspices of the U.S. Department of energy by the Lawrence Livermore National Laboratory under contract No. W-740-ENG-48.

\section{References}

[1] B. Jaskorzynska, J. Nilsson, and L Thylén, J. Opt. Soc. Am B 8, 484 (1991).

[2] P-A Besse, J-S Gu, and H. Melchior, IEEE JQE 27, 1830 (1991); J Buus, M. C. Farries, and D. Robbins, IEEE JQE 27, 1837 (1991); Z. Wang, B. Mikkelsen, and K.E. Stubkjaer, IEEE Photon. Technol. Lett. 3, 366 (1991). 